\title{
Ángulo de pendiente tibial posterior "Slope" en prótesis total de rodilla. Diferencias entre alineación Intra o Extramedular y su repercusión sobre el rango de movilidad postoperatorio.
}

\author{
DOI: http://dx.doi.org//10.37315/SOTOCAV201928054143
}

CRUZADO-NAVARRO B, MIFSUT-MIEDES D, SILVESTRE-MUÑOZ A.

DEPARTAMENTO DE CIRUGÍA UNIVERSIDAD DE VALENCIA

\begin{abstract}
Resumen.
Introducción: El Objetivo del presente trabajo fue comparar la pendiente tibial posterior del componente tibial tras la realización de una Artroplastia total de rodilla, según se utilizó una guía intramedular o extramedular durante la técnica quirúrgica, así como, comparar el rango de movilidad obtenido según la instrumentación utilizada. Material y métodos: Realizamos un estudio descriptivo, retrospectivo, observacional de una serie de 57 pacientes intervenidos en nuestro Centro durante los años 2012 y 2013, con el mismo modelo de Artroplastia total de rodilla (Sigma PS $₫$ DePuy), divididos en dos grupos homogéneos en cuanto a edad, sexo, grado de evolución de la artrosis, el primero con pacientes intervenidos mediante guía extramedular y el segundo mediante guía intramedular. Se realizó un estudio radiográfico, midiendo en el plano sagital el ángulo de pendiente posterior. Se estudió el rango de movilidad alcanzado tras la artroplastia y la supervivencia del implante. Resultados: De forma conjunta, en ambos grupos se realizaron mediciones del ángulo de pendiente posterior, que fue de $4,35^{\circ}$ de media preoperatorio. Por grupos, la media del ángulo de caída posterior postoperatorio fue de $4,04^{\circ}$ en los pacientes intervenidos mediante guía EM, mientras los intervenidos con guía IM la media fue de $1,76^{\circ}$; siendo las diferencias estadísticamente significativas. El rango de movilidad en las ATR intervenidas mediante guía intramedular, fue de $102,7^{\circ}$ de media (rango $80-125^{\circ}$ ), mientras que en el grupo donde se utilizó la guía extramedular, fue de $104,3^{\circ}$ (rango $80-130^{\circ}$ ) no siendo estas diferencias estadísticamente significativas. En cuanto a la supervivencia del implante, durante el periodo de estudio se encontraron dos casos de pacientes intervenidos para el recambio de prótesis, ambos pertenecían al grupo "Guía extramedular". Conclusión: El ángulo de pendiente tibial posterior, para un mismo modelo de prótesis PS, fue diferente estadísticamente según la guía utilizada, encontrándose dentro del rango $3-7^{\circ}$ en el grupo donde se utilizó la guía extramedular; pero a pesar de estas diferencias, no se observó repercusión sobre el rango de movilidad. Actualmente, se recomienda restablecer la pendiente tibial posterior propia del paciente, y en los casos con una pendiente mayor de $10^{\circ}$ utilizar una ATR PS.
\end{abstract}

PALABRAS CLAVE: Total knee arthroplasty; Knee instability; Knee reconstruction; Extramedullary; Intramedullary; MetaAnalysis, slope.

\section{Summary.}

Introduction: The aim of this work was to compare the posterior tibial slope of the tibial component after performing a total knee arthroplasty, as an intramedullary or extramedullary guide was used during the surgical technique, as well as comparing the range of mobility obtained according to the Instrumentation used. Material and methods: We conducted a descriptive, retrospective, observational study of a series of 57 patients operated in our Center during 2012 and 2013 , with the same model of total knee arthroplasty (Sigma PS® DePuy), divided into two homogeneous groups in terms of age, sex, degree of osteoarthritis evolution, the first with patients operated by extramedullary guidance and the second by intramedullary guidance. A radiographic study was performed, measuring the posterior slope angle in the sagittal plane. The range of mobility achieved after arthroplasty and implant survival was studied. Results: Together, in both groups, measurements of the posterior slope angle were made, which was $4.35^{\circ}$ preoperative average. In groups, the mean postoperative posterior fall angle was $4.04^{\circ}$ in the patients who underwent an EM guide, while those who underwent an IM guide the mean was $1.76^{\circ}$; the differences being statistically significant. The range of mobility in the ATRs operated by intramedullary guidance was $102.7^{\circ}$ on average (range $80-125^{\circ}$ ), while in the group where the extramedullary guide was used, it was $104.3^{\circ}$ (range $80-130^{\circ}$ ) no these differences being statistically significant. Regarding the survival of the implant, during the study period two cases of patients undergoing surgery for the replacement of prostheses were found, both of which belonged to the "Extramedullary guide" group. Conclusion: The posterior drop angle, for the same PS prosthesis model, was statistically different according to the guide used, being within the 3-70 range in the group where the extramedullary guide was used; but despite these differences, no impact on the range of mobility was observed. Currently, it is recommended to restore the patient's posterior tibial slope, and in cases with a slope greater than $10^{\circ}$ use a PS ATR. 
CRUZADO B, Y COLS. Ángulo de pendiente tibial posterior "Slope" en prótesis total de rodilla. Diferencias entre alineación Intra o Extramedular y su repercusión sobre el rango de movilidad postoperatorio.

Correspondencia:

Dr. Damián Mifsut Miedes

mifsut_dam@gva.es

\section{Introducción}

Existen varios factores que pueden afectar al rango de movilidad tras una artroplastia total de rodilla (ATR), incluida la movilidad previa, la técnica quirúrgica, la fisioterapia y el diseño del implante, entre otros ${ }^{1-4}$.

Cuando consideramos la pendiente tibial posterior (Slope) en el plano sagital, muchos estudios han demostrado que lograr una adecuada pendiente posterior es crucial para la movilidad y estabilidad de la ATR.

Generalmente se cree que un aumento de la pendiente tibial posterior puede mejorar en gran medida la flexión máxima postoperatoria de la rodilla. Catani y cols. ${ }^{5}$, así como, Malviva y Cols. ${ }^{6}$, informaron que un aumento de la pendiente tibial posterior in vivo puede mejorar la flexión máxima tras una ATR con preservación del ligamento cruzado posterior (CR)

Sin embargo, esto no está claramente demostrado en los estudios clínicos, los cuales no respaldan la correlación positiva entre la pendiente tibial y la flexión máxima de la rodilla ${ }^{4,7-13}$

También, el incremento de la pendiente tibial posterior influirá sobre la flexión de forma diferente dependiendo del modelo de prótesis, estabilizada posterior (PS) o con retención del LCP (CR).

El Objetivo del presente trabajo fue comparar la pendiente tibial posterior del componente tibial tras la realización de una Artroplastia total de rodilla, según se utilizó una guía intramedular o extramedular durante la técnica quirúrgica, así como, comparar el rango de movilidad obtenido según la instrumentación utilizada.

\section{Material y Métodos}

Realizamos un estudio descriptivo, retrospectivo, observacional de una serie de 57 pacientes intervenidos en nuestro Centro durante los años 2012 y 2013, de Artroplastia total de rodilla.

Se establecieron dos grupos:

- $\quad$ el primer grupo constó de 28 casos intervenidos mediante guía extramedular; intramedular.

el segundo con 29 casos intervenidos con guía

Todos los casos de cada grupo fueron intervenidos por el mismo cirujano y con el mismo modelo de prótesis: Sigma de De Puy®, de tipo estabilizada posterior, en ambos. El tiempo de seguimiento mínimo fue de 6 años.

Los pacientes fueron seleccionados consecutivamente según el orden de intervención.
Además de las variables demográficas, se estudió el grado de artrosis según la clasificación de Ahlbäck, el rango de movilidad alcanzado y la supervivencia del implante.

Se realizó un estudio radiográfico, midiendo en el plano sagital el ángulo de pendiente tibial posterior descrito por Dejour $^{14}$.

Se localizó el punto central distal a la tuberosidad y el punto central diafisario más caudal que nos permitía la radiografía como eje tibial, midiendo el ángulo formado por éste eje con respecto a la pendiente tibial posterior (Fig.1).

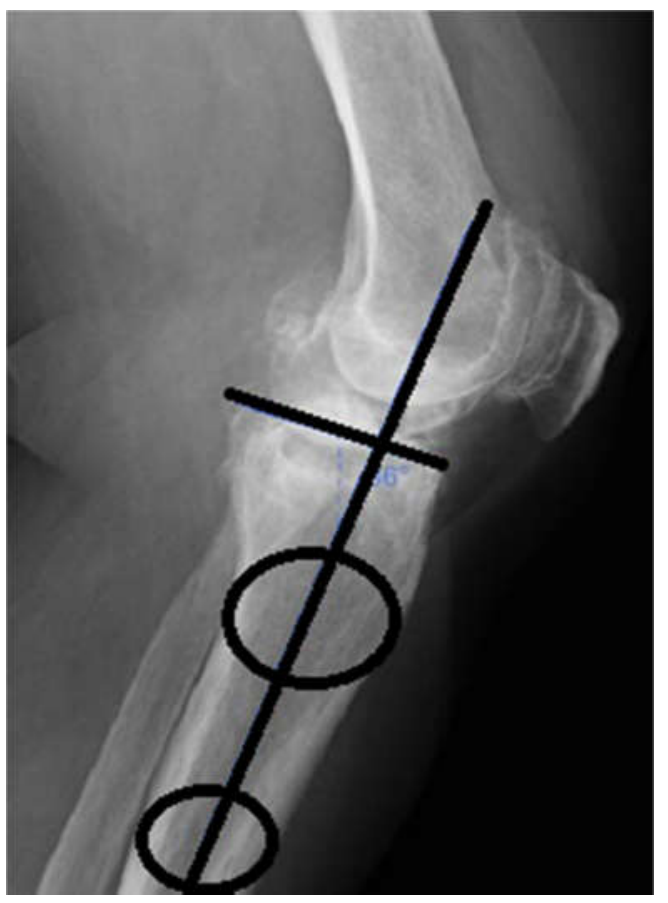

Figura 1. Eje Diafisario Sagital Proximal Descrito por Dejour /Caída Posterior/ Pendiente Tibial.

Para el análisis estadístico, se utilizó el programa estadístico informático IBM SPSS Statistics 25, realizándose un estudio descriptivo de las diferentes variables, y comparándose el ángulo de la pendiente posterior entre ambos grupos mediante la Prueba de Chi-Cuadrado. La correlación entre el rango de movilidad y dicho ángulo entre los dos grupos se realizó con pruebas no paramétricas (Tau_b de Kendall).

\section{Resultados}

Del total de 57 pacientes, 16 eran hombres (28\%) y 41 mujeres(72\%), con una distribución similar en ambos grupos: 21 mujeres en el grupo intervenido mediante 
CRUZADO B, Y COLS. Ángulo de pendiente tibial posterior "Slope" en prótesis total de rodilla. Diferencias entre alineación Intra o Extramedular y su repercusión sobre el rango de movilidad postoperatorio.

guía intramedular y 20 en el grupo de guía extramedular, 8 hombres en cada grupo. Existió un claro predominio del sexo femenino 41, frente a 16 hombres.

La media de edad de los pacientes intervenidos fue de 77,23 años (rango 61-88).

En cuanto al lado o pierna intervenida, la mayoría de las ATR fueron de lado derecho, $32(56,14 \%)$ frente a 25 $(43,86 \%)$ del lado izquierdo, y la distribución en ambos grupos fue: en las guías extramedulares (grupo l) 19 del lado derecho $(67,86 \%)$ y 9 del lado izquierdo $(32,14 \%)$; en las guías intramedulares (grupo II), 13 del lado derecho $(44,83 \%)$ y 16 del izquierdo $(55,17 \%)$.

Según la Clasificación de Ahlbäck, la mayoría (35 casos) fueron clasificadas como Grado IV, y en 20 casos, Grado III (Tabla VIII). En el Grupo I (extramedulares) 18 casos fueron grado IV, 9 grado III y 1 grado II; y en el grupo II (intramedulares), 17 grado IV, 11 grado III y 1 grado II. Por tanto, ambos grupos eran homogéneos en cuanto al sexo, edad y grado de artrosis según la clasificación de Ahlbäck (Tabla I).

\begin{tabular}{lll|l|l|l} 
& & & & & Porcentaje \\
& & Frec & $\%$ & $\%$ Val & acumulado \\
\hline Vál 2 & 2 & 3,5 & 3,5 & 3,5 \\
\cline { 2 - 5 } ido 3 & 20 & 35,1 & 35,1 & 38,6 \\
\hline 4 & 35 & 61,4 & 61,4 & 100,0 \\
\hline $\begin{array}{c}\text { To } \\
\text { tal }\end{array}$ & 57 & 100,0 & 100,0 & \\
\hline
\end{tabular}

Tabla I. Grado de artrosis según la clasificación de Ahlbäck .

La gran mayoría de los casos 49/57 (86\%), presentaban una deformidad de su rodilla en varo, distribuidos de forma similar en ambos grupos, 23 en el grupo I y 26 en el grupo II. De forma conjunta, en ambos grupos se realizaron mediciones del ángulo de pendiente posterior, que fue de $4,35^{\circ}$ de media preoperatorio.

En las mediciones postoperatorias el ángulo de pendiente posterior pasó a un ángulo de $2,83^{\circ}$ de media (Tabla II).

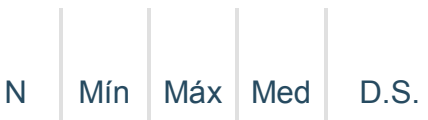

\begin{tabular}{|c|c|c|c|c|c|}
\hline C. AHLBÄCK & 57 & 2 & 4 & 3,58 &, 565 \\
\hline $\begin{array}{l}\text { M.PREQX } \\
\text { PENDIENTE } \\
\text { POST }\end{array}$ & 57 & 80 & 90 & $\begin{array}{r}85,6 \\
5\end{array}$ & 2,622 \\
\hline $\begin{array}{l}\text { M.POSTQX } \\
\text { PENDIENTE } \\
\text { POST }\end{array}$ & 57 & 81 & 90 & $\begin{array}{r}87,1 \\
2\end{array}$ & 2,323 \\
\hline $\begin{array}{l}\text { Diferencia } \\
\text { pre-postQ } \\
\text { Slope }\end{array}$ & 57 & -9 & 9 & 1,49 & 3,689 \\
\hline
\end{tabular}

Tabla II. Grado de artrosis y valores del ángulo de pendiente posterior pre y postquirúrgico de toda la serie (ángulos complementarios a $90^{\circ}$ ).

Por grupos, la media del ángulo de caída posterior postoperatorio fue de $4,04^{\circ}$ en los pacientes intervenidos mediante guía EM, mientras los intervenidos con guía IM la media fue de $1,76^{\circ}$; siendo las diferencias estadísticamente significativas. (Tabla III).

\begin{tabular}{lr|r|r} 
& & & $\begin{array}{c}\text { Significac } \\
\text { ión } \\
\text { asintótica } \\
\text { (bilateral) }\end{array}$ \\
\hline $\begin{array}{l}\text { Chi-cuadrado de } \\
\text { Pearson }\end{array}$ & $\begin{array}{r}16,94 \\
\text { df }\end{array}$ & 7 &, 018 \\
\hline Razón de & 21,45 & 7 &, 003 \\
verosimilitud & 4 & & \\
\hline Asociación lineal & 13,69 & 1 &, 000 \\
por lineal & 1 & & \\
\hline N de casos & 57 & & \\
válidos & & & \\
\hline
\end{tabular}


CRUZADO B, Y COLS. Ángulo de pendiente tibial posterior "Slope" en prótesis total de rodilla. Diferencias entre alineación Intra o Extramedular y su repercusión sobre el rango de movilidad postoperatorio.

Tabla III. Prueba de chi-cuadrado para el ángulo de pendiente posterior.

El rango de movilidad en las ATR intervenidas mediante guía intramedular, fue de $102,7^{\circ}$ de media (rango 80 $\left.125^{\circ}\right)$, mientras que en el grupo donde se utilizó la guía extramedular, fue de $104,3^{\circ}$ (rango $80-130^{\circ}$ ) no siendo estas diferencias estadísticamente significativas.

En cuanto a la supervivencia del implante, durante el periodo de estudio se encontraron dos casos de pacientes intervenidos para el recambio de prótesis, ambos pertenecían al grupo "Guía extramedular".

\section{Discusión}

Actualmente no existe consenso en cuanto al nivel de resección de pendiente tibial posterior, aunque la mayoría de autores aconseja entre $3^{\circ}$ y $7^{\circ}$.

Para conseguir reproducir el plano de corte tibial, tanto las guías extramedulares como las intramedulares parecen ser adecuadas. Cuando utilizamos una guía extramedular, buscamos a nivel distal el punto medio de la distancia entre ambos maléolos del tobillo, y utilizamos una mayor o menos separación de la barra respecto a la cresta tibial, de modo que cuando mayor sea la distancia entre la barra y la tibia a nivel distal, mayor será la pendiente posterior del corte tibial.

Según Maestro y Cols. $^{15}$, Los pacientes con deformidades extraarticulares significativas, arqueamiento marcado y aquellos con cirugía previa o fracturas pueden no ser adecuados para guías intramedulares, y pueden requerir el uso de guías extramedulares y control radiográfico intraoperatorio. La indicación ideal para el uso de instrumentación intramedular es en el paciente que no es obeso, sin deformidad extraarticular y con un canal medular tibial bien definido, pero no excesivamente ancho. Dado que la mala alineación del componente tibial en general, y la mala alineación del plano coronal en particular, pueden afectar negativamente la supervivencia a largo plazo de las artroplastias totales de rodilla, se recomienda el uso de instrumentación de alineación intramedular cuando sea posible.

Normalmente, se recomienda hacer coincidir la pendiente posterior protésica con la pendiente tibial original del paciente ${ }^{16}$, por lo que la guía intramedular puede reproducir con mayor exactitud el grado de pendiente posterior elegido.

De Kroon y Cols. ${ }^{13}$ llevaron a cabo un ECA de dos brazos, reclutando pacientes que se sometieron a ATR mínimamente invasiva. Los resultados de interés en el estudio fueron el ángulo tibio-femoral y la pendiente tibial. No se encontraron diferencias significativas para los resultados en el plano coronal. Hubo una diferencia significativa en la restauración de la pendiente tibial con el grupo EM que tenía una pendiente restaurada más cerca de los valores preoperatorios $(P=0.001)$. En nuestro trabajo también encontramos diferencias E.S. en el mismo sentido, la guía extramedular mantenía mejor la pendiente posterior entre 3 y $7^{\circ}$.

Sin embardo, esto no tuvo ninguna repercusión sobre el rango de movilidad final, ya que no se observaron diferencias significativas entre ambos grupos.

Karade y Cols. ${ }^{17}$, no encontraron diferencias al utilizar guía intra o extrarticular en la implantación de megaprotesis en los planos coronal y sagital.

Hernigou y Deschamps ${ }^{18}$, en un estudio con prótesis unicompartimentales encontraron que una pendiente tibial menor de $7^{\circ}$, reduce el riesgo de lesión o potencial rotura del LCA. Este estudio también mostraba que un incremento de la pendiente se correlacionaba con un incremento de la traslación tibial durante la carga. Dejour y Bonnin ${ }^{14}$, vieron que cada incremento de $10^{\circ}$ de la pendiente tibial posterior en apoyo monopodal se asocia con un incremento adicional de $6 \mathrm{~mm}$. de traslación tibial anterior, siendo la traslación más pronunciada en casos de déficit o ausencia de LCA.

En la mayoría de casos, el inserto de polietileno tibial de una artroplastia unicompartimental es plano y por lo tanto el movimiento no tiene restricciones, por lo tanto, los resultados variarán con insertos más conformados ${ }^{16}$.

Sin embargo, Hernigou y Deschamps refieren que el incremento de la pendiente posterior tibial puede causar una significativa traslación tibial incluso con el LCA intacto. Estos autores mantienen que una pendiente tibial posterior óptima se sitúa entre $3^{\circ}$ y $7^{\circ}{ }^{18}$.

Garg y Walker ${ }^{19}$ refieren que el incremento de la pendiente posterior favorece la flexión y el rollback femoral. También conduce a mejorar la distribución de las cargas en el interfaz hueso-implante, consiguiendo el mejor grado de movilidad con una pendiente posterior de $10^{\circ}$.

Shi $X$ y Cols. ${ }^{20}$ mostraron que cada incremento de $1^{\circ}$ de pendiente posterior, se incrementaba en $1,8^{\circ}$ la flexión en una ATR PS.

Ken Okazaki y Cols. ${ }^{21}$, observaron intraoperatoriamente que, con cambios en la pendiente posterior de $5^{\circ}$, el gap en flexión cambió aproximadamente $2 \mathrm{~mm}$ con una ATR $\mathrm{CR}$, siendo de $1 \mathrm{~mm}$ con una ATR PS, es decir, un cambio de pendiente posterior influye más en el Gap en flexión con una ATR-CR que con ATR-PS.

Nowakowski y Cols. ${ }^{22}$, concluyeron en su trabajo que "un incremento de la pendiente tibial posterior más allá del planificado preoperatoriamente, para conseguir ampliar un gap estrecho intraoperatoriamente no está recomendado, ya que al hacerlo aumentará también el gap en extensión, pudiendo también desequilibrar de medial y lateral el gap en flexión". Esto puedo provocar una inestabilidad anterior o mayor laxitud en flexión media; por lo tanto el equilibrado debe hacerse sobre partes blandas para conseguir un adecuado balance ligamentoso, sin mayores resecciones óseas. 
CRUZADO B, Y COLS. Ángulo de pendiente tibial posterior "Slope" en prótesis total de rodilla. Diferencias entre alineación Intra o Extramedular y su repercusión sobre el rango de movilidad postoperatorio.

El ángulo de inclinación tibial posterior en prótesis $\mathrm{CR}$ debe reproducir la pendiente previa para conseguir una adecuada tensión del LCP, pero cuando ésta pendiente previa es mayor de $10^{\circ}$ existen dos posibilidades: continuar reproduciendo la pendiente propia del paciente o realizar el corte con una pendiente estándar $\left(3-5^{\circ}\right)$.

En el primer caso, al mantener la inclinación propia del paciente (mayor de $10^{\circ}$ ) la tensión y orientación del LCP será la adecuada, pero puede aumentar el riesgo de subluxación anterior del componente tibial a la carga y mayor desgaste del polietileno a nivel posterior ${ }^{23}$.

Si se realiza un corte estándar $\left(3-5^{\circ}\right)$ corremos el riesgo de perder flexión en la rodilla y aumentar la presión sobre la parte posterior y por tanto mayor desgaste del polietileno en dicha zona.
Por ello, se recomienda utilizar una ATR PS en los pacientes con deformidad en flexo mayor de $15^{\circ}$ y en mesetas tibiales con una pendiente posterior mayor de $10^{\circ}$.

\section{Conclusiones}

El ángulo de pendiente tibial posterior, para un mismo modelo de prótesis PS, fue diferente estadísticamente según la guía utilizada, encontrándose dentro del rango 3-7 $7^{\circ}$ en el grupo donde se utilizó la guía extramedular; pero a pesar de estas diferencias, no se observó repercusión sobre el rango de movilidad.

Actualmente, se recomienda restablecer la pendiente tibial posterior propia del paciente, y en los casos con una pendiente mayor de $10^{\circ}$ utilizar una ATR PS. 
CRUZADO B, Y COLS. Ángulo de pendiente tibial posterior "Slope" en prótesis total de rodilla. Diferencias entre alineación Intra o Extramedular y su repercusión sobre el rango de movilidad postoperatorio.

\section{Bibliografía}

1. Kang K, Jang YW, Yoo OS, Jung D, Lee SJ, Lee MC, et al. Biomechanical Characteristics of Three Baseplate Rotational Arrangement Techniques in Total Knee Arthroplasty. Biomed Res Int 2018; 2018. https://doi.org/10.1155/2018/9641417

2. Feeley I, Hegarty A, Hickey A, Glynn A. Impact of Use of Intramedullary and Extramedullary Guides on Tibial Component Geometry in Total Knee Replacements: A Systematic Review and Meta-Analysis. J Knee Surg 2016;29(6):487-96.

3. D'Lima D, Hermida C, Chen P, Colwell C. Polyethylene wear and variations in knee kinematics. Clin Orthop 2001; 392:124-30.

4. Dennis DA, Channer M, Susman MH, Stringer EA. Intramedullary versus extramedullary tibial alignment systems in total knee arthroplasty. J Arthroplasty 1993; 8(1):43-7.

5. Catani F, Fantozzi S, Ensini A, Leardini A, Moschella D, Giannini S.. Influence of tibial component posterior slope on in vivo knee kinematics in fixed-bearing total knee arthroplasty. J Orthop Res. 2006 Apr;24(4):581-7.

6. Malviya A1, Lingard EA, Weir DJ, Deehan DJ. Predicting range of movement after knee replacement: the importance of posterior condylar offset and tibial slope. Knee Surg Sports Traumatol Arthrosc. 2009 May;17(5):491-8. doi: 10.1007/s00167-008-0712-x.

7. Jevsevar DS. Treatment of osteoarthritis of the knee: Evidence-based guideline, 2nd edition. J Am Acad Orthop Surg 2013; 21(9):571-6.

8. $\mathrm{Li} \mathrm{Y,} \mathrm{Li} \mathrm{A,} \mathrm{Zhang} \mathrm{Y.} \mathrm{The} \mathrm{efficacy} \mathrm{of} \mathrm{combined} \mathrm{adductor} \mathrm{canal} \mathrm{block} \mathrm{with} \mathrm{local} \mathrm{infiltration} \mathrm{analgesia} \mathrm{for} \mathrm{pain} \mathrm{control}$ after total knee arthroplasty: A meta-analysis. Medicine (Baltimore) 2018; 97(49):e13326.

9. Mahaluxmivala J, Bankes MJ, Nicolai P, Adlam CH, Allen PW. The effect of surgeon experience on component posi- tioning in 673 press fit condylar posterior cruciate-sacrificing total knee arthroplasties. J Arthroplasty 2001; 16:635-40.

10. Insall JN, Binazzi R, Soudry M, Mestriner LA. Total knee arthroplasty. Clin Orthop Relat Res 1985;192: 13-22.

11. Ritter MA, Davis KE, Meding JB, Pierson JL, Berend ME, Malinzak RA. The effect of alignment and BMI on failure of total knee replacement. J Bone Joint Surg Am 2011; 93(17):1588-96.

12. Matsuda S, Whiteside L, White $S$. The effect of varus tilt on contact stress in total knee arthroplasty. Orthopedics 1999; 22:303-7.

13. De Kroon KE, Houterman S, Janssen RPA. Leg alignment and tibial slope after minimal invasive total knee arthroplasty: a prospective, randomized radiological study of intramedullary versus extramedullary tibial instrumentation. Knee 2012; 19(4):270-4.

14. Dejour $\mathrm{H}$, Bonnin $\mathrm{M}$. Tibial translation after anterior cruciate ligament rupture. Two radiological tests compared. $\mathrm{J}$ Bone Joint Surg Br 1994 Sep; 76(5):745-9.

15. Maestro A, Harwin SF, Sandoval MG, Vaquero DH, Murcia A: Influence of intramedullary versus extramedullary alignment guides on final total knee arthroplasty component position: a radiographic analysis. J Arthroplasty 1998; 13(5):552-8.

16. Fehring TK, Odum S, Griffin WL, Mason JB, Nadaud M. Early failures in total knee arthroplasty. Clin Orthop Relat Res $2001 ; 392: 315-8$.

17. Karade V, Ravi B, Agarwal M. Extramedullary versus intramedullary tibial cutting guides in megaprosthetic total knee replacement. J Orthop Surg Res 2012; 2(7):33. doi: 10.1186/1749-799X-7-33.

18. Hernigou P1, Deschamps G. Posterior slope of the tibial implant and the outcome of unicompartmental knee arthroplasty. J Bone Joint Surg Am 2004 Mar; 86(3):506-11.

19. Garg A, Walker PS. Prediction of total knee motion using a three-dimensional computer-graphics model. J Biomech 1990;23(1):45-58.

20. Shi X, Shen B, Pengde K, Yang J, Zhou Z, Pei F. The effect of posterior tibial slope on knee flexion in posteriorstabilized total knee arthroplasty. Knee Surgery, Sports Traumatology, Arthroscopy 2013; 21:2696-703.

21. Okazaki K, Tashiro, Mizu-uchi HY, Hamai S, Doi T, Iwamoto Y. Influence of the posterior tibial slope on the flexion gap in total knee arthroplasty. Journal of Arthroplasty 2015; 30(8): 1439-1443. DOI: 10.1016/j.knee.2014.02.019

22. Nowakowski AM1, Kamphausen M, Pagenstert G, Valderrabano V, Müller-Gerbl M. Influence of tibial slope on extension and flexion gaps in total knee arthroplasty: increasing the tibial slope affects both gaps. Int Orthop 2014; 38(10):2071-7. doi: 10.1007/s00264-014-2373-3.

23. Migaud H1, De Ladoucette A, Dohin B, Cloutier JM, Gougeon F, Duquennoy A. [Influence of the tibial slope on tibial translation and mobility of non-constrained total knee prosthesis]. Rev Chir Orthop Reparatrice Appar Mot 1996; 82(1):7-13. 